\title{
Evaluation of perceived quality attributes of digital holograms viewed with a stereoscopic display
}

\author{
(Invited paper)
}

\author{
Taina M. Lehtimäki, Kirsti Sääskilahti, Tomi Pitkäaho \\ Oulu Southern Institute \\ University of Oulu \\ 84100 Ylivieska, Finland
}

\author{
Thomas J. Naughton \\ Department of Computer Science \\ National University of Ireland, Maynooth, Ireland, and \\ Oulu Southern Institute, University of Oulu \\ 84100 Ylivieska, Finland \\ tomn@cs.nuim.ie
}

\begin{abstract}
Holography is a well-known technique for sensing and displaying real-world three-dimensional (3D) objects. Digital holograms have been successfully displayed on conventional stereoscopic displays allowing research into perception of quality of 3D holographic data. We do know that quality is enhanced if reconstructions of digital holograms are displayed with conventional stereoscopic displays rather than with a regular two-dimensional (2D) screen. However, it is not known how different attributes (e.g. noise, blur, and perceived depth) and the viewer's subjective perception of quality are related. In this study, we show how 13 viewers evaluated these attributes and the visual quality of five holograms displayed stereoscopically.
\end{abstract}

\section{INTRODUCTION}

In digital holography $[1,2,3]$ we capture optically a 3D scene and reconstruct the desired perspectives numerically in a computer. The reconstructions are routinely displayed in the form of a 2D image slice through the reconstruction volume, an extended focus image, or a depth map from a single perspective. These are fundamentally $2 \mathrm{D}$ (or at most $2 \frac{1}{2} \mathrm{D}$ ) representations and are not certain to give the human viewer a clear perception of the 3D features encoded in the hologram. Alternatively, an optical reconstruction would allow the human viewer to extract the 3D information themselves through stereopsis, motion parallax, blur, and so on, but convincing full-field digital holographic displays for real-world 3D scenes have not been demonstrated due to current limitations of programmable display devices.

As an intermediate measure, we have digitally processed the holograms to display them on conventional stereoscopic displays, which provide a readily understood and off-the-shelf available technology, and the human visual system itself extracts the 3D information of the scene encoded in the hologram. Previously we have reported results suggesting that when digital hologram reconstructions are displayed stereoscopically people perceive less speckle noise and increased depth of field $[4,5]$ and how displaying the reconstructions in stereo reduces the perception of noise, increases the detail visibility and general enhances perceived quality when compared to displaying the reconstructions as 2D images $[5,6]$. This paper reports a continuation to this research.

There are methods concerning image quality measurement of stereoscopic images [7, 8, 9]. In greyscale images the distortions related to the noise, blur and speckle are the essential part of the image quality. Quality evaluations made with conventional images with additional distortions may even improve the subjectively perceived quality (edge enhancement, noise addition) [10]. A previous study has evaluated how viewers perceive depth in a single-view video reconstruction from a holographic dynamic 3D scene [11]. The effects of hologram compression on reconstruction quality for dynamic 3D scenes has been investigated [12]. The increased depth of field that accompanies disbalanced stereo display of hologram reconstructions at different depths has been studied [13].

In this paper we look into three different attributes (noise, blur, and perceived depth) contributing to the perceived quality of a stereoscopic image and show in detail results of how a group of viewers evaluated the visual quality of five holograms displayed stereoscopically with different reconstruction window sizes.

\section{METHOD}

\section{A. Subjects and apparatus}

Our experiments were performed using 13 viewers with normal or corrected-to-normal vision (ten females and three males varying in age between 23 and 53). Their stereovision was tested with stereo pair photographs before the experiment to ensure they had good stereovision acuity. Stimuli were presented on a Sharp LL-151-3D LCD monitor. This is an autostereoscopic display; it allows users to see stereoscopic images without using special glasses. It consists of two LCD panels, one in front of another. In 3D mode the foreground LCD panel acts as a parallax barrier so that when the head is positioned appropriately, the right and left eyes of a viewer receive different images. The screen resolution was $1024 \times 768$ pixels ( $512 \times 768$ pixels for each eye) and the distance between the subject and screen was approximately $60 \mathrm{~cm}$. 

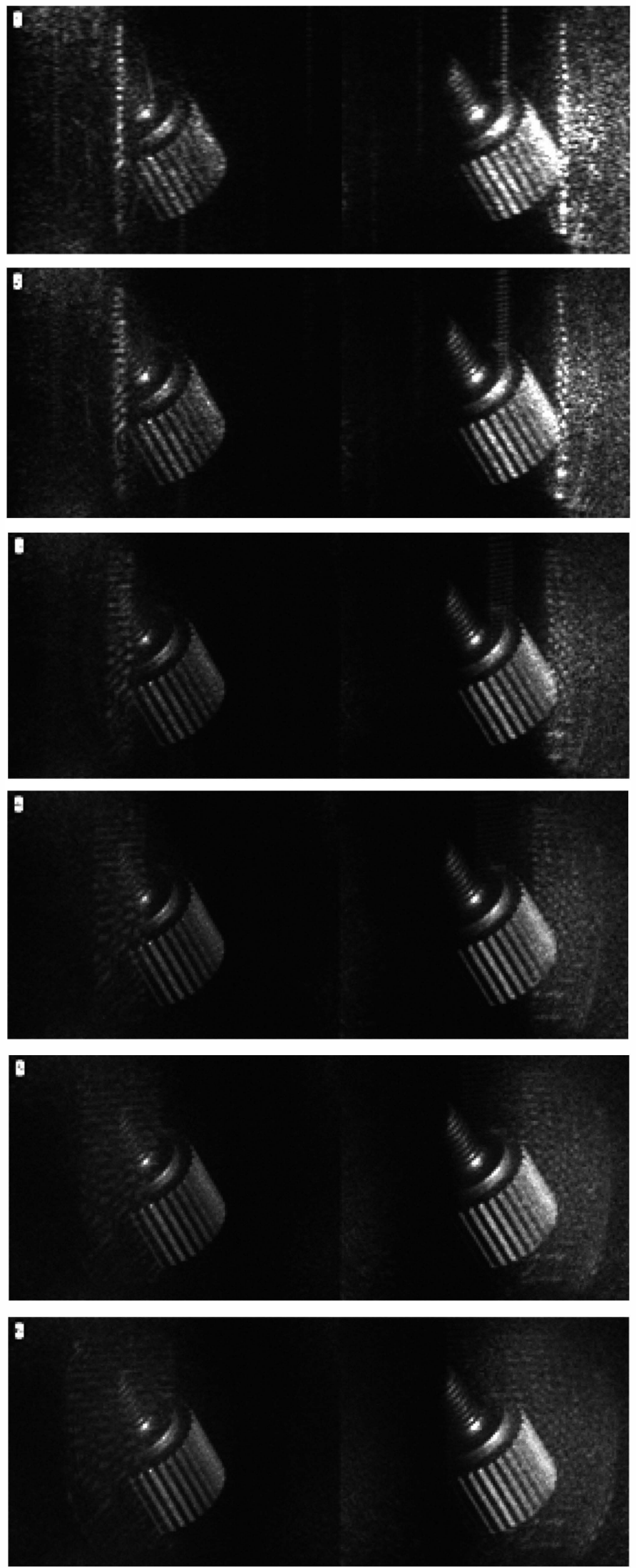

Figure 1. Left and right reconstructions for the stereo stimuli of the Hologram 2 from window size $64 \times 64$ (top) to $1024 \times 1024$ pixels (bottom).

\section{B. Stimuli}

The holograms were captured of real-world objects with side lengths of $1 \mathrm{~cm}$ and reconstructed using simulated Fresnel propagation [14]. We used a set of reconstructed stereopairs from each of five digital holograms (Hologram 1 - Hologram 5). Each hologram has its own reconstruction depth where the whole object was perceived in focus. Each set of stereopairs was reconstructed using six different hologram window (aperture) sizes starting from size $64 \times 64$ pixels up to size $1024 \times 1024$ pixels. The complete set of window side lengths used were $64,128,256,512,768$ and 1024. A larger hologram window means more pixels are used in the reconstruction resulting in a higher quality image. A common center location for each of the windows admitting left and right reconstructions was chosen resulting in the same perspective for each stereopair independent of window size. An example of the stereopair set of Hologram 2 is presented in Figure 1.

\section{Procedure}

Viewers were asked to evaluate subjectively different attributes regarding the quality of the stereoscopically displayed hologram reconstruction pairs. The participants could browse the set of hologram reconstructions with different window sizes. The images were numbered so that the window size 64 was number one and the window size 1024 was number six. The participants were asked to select for which images of the set: (1) the noise does not disturb, (2) all details of the object can be seen clearly so that blur does not disturb, and (3) there is a clear perception of depth. At the end they were asked to evaluate the quality of each stereoimage using the scale from 1 to 5 (from very poor to very good, respectively).

\section{RESULTS AND CONCLUSIONS}

Figure 2 shows the results for the set of five holograms used in this study. Although there are differences between different holograms, it is clear for each of the five holograms all evaluated attributes act similarly to the evaluated quality grade. There is correlation between the subjectively graded quality of the image and all three attributes. From these we can draw a conclusion that each measured attribute may be an important part of subjectively perceived quality.

However, there are three interesting discontinuations in the general trends in Figure 2: two drops in plots of perception of depth (for Hologram 2 at window size 768 and for Hologram 4 at window size 512), and one in the plot for absence of blur for Hologram 4 at window size 512. We may speculate that if the perception of quality simply depended on one of these attributes, there should also be clear drop in quality graphs of these holograms for the same window sizes. Since this is not the case we conclude that the perceived quality is not solely depended on one of these attributes. The subjective perception of quality of holograms is very complex and this topic needs to be studied in more depth.

From Figure 2 we can also draw a conclusion that quality is strongly enhanced with larger window size up to a window size of 512. However, for some holograms, a larger than 512 window size (i.e. 768 and 1024) does not seem to further enhance the quality. 

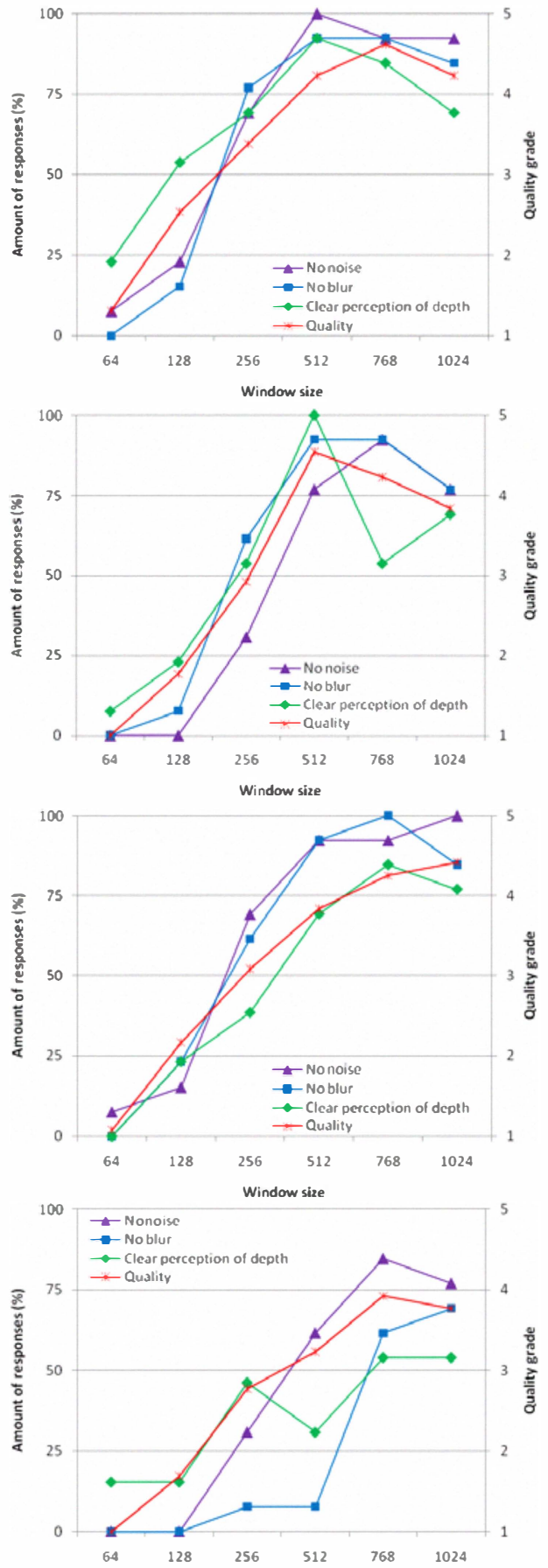

Window size

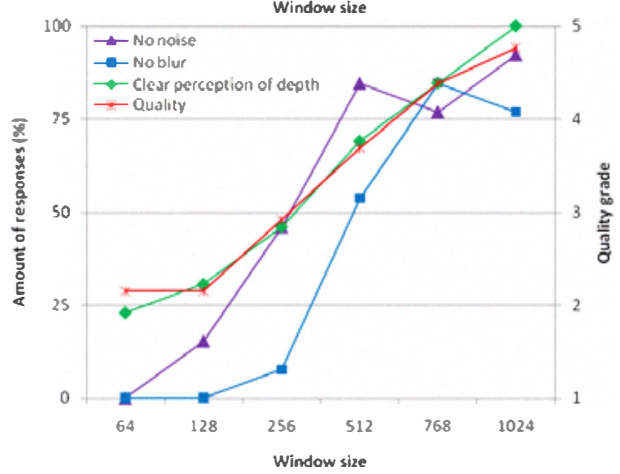

Figure 2. (Shown on the left.) Results for perceived noise, blur, perception of depth, and quality shown separately for each of the five holograms (Hologram 1 on top and Hologram 5 on the bottom). Quality is measured using a scale from 1 to 5 (right vertical axis) and the other attributes are measured as percentage of responses (left vertical axis). The square window size from 64 to 1024 measured in pixels is on the horizontal axis.

\section{ACKNOWLEDGEMENTS}

The research leading to these results has received funding from Science Foundation Ireland under the National Development Plan, the Academy of Finland, and the European Community's Seventh Framework Programme FP7/2007-2013 under Grant agreement No. 216105 (acronym "Real 3D"). Thanks to Conor Mc Elhinney for recording the holograms.

\section{REFERENCES}

[1] D. Gabor, "A new microscopic principle," Nature, vol. 161, pp. 777-778, 1948.

[2] Th. Kreis, Handbook of Holographic Interferometry: Optical and Digital Methods, Wiley, 2005.

[3] Y. Frauel, T.J. Naughton, O. Matoba, E. Tajahuerce, and B. Javidi, "Three-dimensional imaging and processing using computational holographic imaging", Proceedings of the IEEE, vol. 94, pp. 636-653, 2006.

[4] T.M. Lehtimäki and T.J. Naughton, "Stereoscopic viewing of digital holograms of real-world objects", 3DTV-Conference 2007 - Capture, Transmission and Display of $3 D$ Video, article no. 39, Kos, Greece, 7-9 May 2007.

[5] T.M. Lehtimäki, K. Sääskilahti, R. Näsänen, T.J. Naughton. "Visual perception of digital holograms on autostereoscopic displays". Proc. SPIE 7329, art. no. 73290C, 2009.

[6] T.M. Lehtimäki, K. Sääskilahti, R. Näsänen, T.J. Naughton., "Stereo perception of reconstructions of digital holograms of real-world objects" Phys.: Conf. Ser. 206, article no. 012030, 2010.

[7] Recommendation ITU-R Rec BT.500-11. Methodology for the subjective Assessment of the Quality of Television Pictures, 2000.

[8] D. Gorley and N. Holliman, "Stereoscopic image quality metrics and compression," Proceedings of SPIE-IS\&T Electronic Imaging, vol. 6803, pp. 680305.1-680305.12, 2008.

[9] J. Häkkinen, T. Kawai, J. Takatalo, T. Leisti, J. Radun, A. Hirsaho and. G. Nyman. "Measuring stereoscopic image quality experience with interpretation based quality methodology," Proceedings. of SPIE-IS\&T Electronic Imaging, vol. 6808, pp. 68081B-68081B-12, 2008.

[10] J. Boyle, A.S. Maeder, and W. Boles, "Visual perception of low quality image," Proceedings of the 9th International Conference on Neural Information Processing, vol. 1, pp. 153-157, 2006.

[11] M. Paturzo, P. Memmolo, A. Finizio, R. Näsänen, T.J. Naughton, and P. Ferraro, "Synthesis and display of dynamic holographic 3D scenes with real-world objects," Optics Express, vol. 18, no. 9, pp. 8806-8815, 2010.

[12] E. Darakis, M. Kowiel, R. Näsänen, and T.J. Naughton, "Visually lossless compression of digital hologram sequences," IS\&T/SPIE Electronic Imaging, Proc. SPIE vol. 7529, article no. 752912, 2010.

[13] R. Näsänen, A. Metso, T.M. Lehtimäki, and T.J. Naughton, "Perceptual quality of reconstructions of digital holograms: extending depth of focus by binocular fusion," Perception, Supplement (32nd European Conference on Visual Perception, Regensburg, Germany, 24-28 August 2009), vol. 38, pp. 154-155, 2009.

[14] C. P. Mc Elhinney, B. M. Hennelly, and T. J. Naughton, "Extended focused imaging for digital holograms of macroscopic three-dimensional objects," Applied Optics, vol. 47, pp. D71-D79, 2008. 